\title{
Sensory Responses in Solution vs. Solid State: A Fluorescence Quenching Study of Poly(iptycenebutadiynylene)s
}

\section{Supporting Information}

\author{
Dahui Zhao and Timothy M. Swager* \\ Department of Chemistry, Massachusetts Institute of Technology, Cambridge, Massachusetts 02139. \\ tswager@mit.edu
}

General Methods ${ }^{1} \mathrm{H}$ NMR spectra were recorded on a Varian Mercury-300 MHz or a Varian Unity$500 \mathrm{MHz}$ instrument. Polymer molecular weights were determined by gel permeation chromatography using an HP series $1100 \mathrm{GPC}$ system running at a flow rate at $1.0 \mathrm{~mL} / \mathrm{min}$ in $\mathrm{THF}$ and a diode detector. The molecular weights are reported relative the polystyrene standards purchased from Polysciences. UV-vis spectra were recorded with an Agilent 8453 diode-array or Cary 50 UV-vis spectrophotometer, corrected for baseline with a solvent-filled cuvette. Emission spectra were acquired on a SPEX Fluorolog- $\tau 3$ fluorometer. The spectra in solution were obtained at room temperature using a quartz cuvette with a path length of $1 \mathrm{~cm}$ at right angle detection. The absorbance OD of all samples used for fluorescence studies were equal to or below 0.1. Polymer thin films were prepared by spin casting a solution of the polymer in chloroform onto a cover glass. Polymer thin films deposited on the interior glass capillaries (for Fido experiments) were prepared as follows. A solution of polymer in chloroform (20-30 $\mu \mathrm{L})$ was injected into a glass capillary $(8 \mathrm{~cm}$ long, ID=0.6 mm) via a syringe. The glass capillary was then attached to the spin-coater platform and the spin-coater was operated at $700 \mathrm{rpm}$ for 1 minute to generate the polymer coated capillary. Fluorescence quantum yields of polymers were determined relative to quinine sulfate $\left(\Phi_{\mathrm{F}}=0.53\right.$ in $0.1 \mathrm{~N}$ sulfuric acid). The lifetimes were determined by phasemodulation method using 10 frequencies between 10 to $200 \mathrm{MHz}$. 
Materials All solvents used in spectroscopy studies in solutions and thin film preparations are spectroscopy grade purchased from Aldrich unless otherwise noted. Anhydrous diisopropylamine was purchased from Aldrich. All other compounds are purchased from Aldrich and used as received. All air- and moisture-sensitive synthetic procedures were performed under argon atmosphere.

\section{Polymer Syntheses.}

General Procedure. A general procedure is illustrated by the synthesis of P1. M1 (110 mg, 0.125 mmol), 1,4-benzoquinone (17 mg, $0.16 \mathrm{mmol}), \mathrm{CuI}(8 \mathrm{mg})$, and $\mathrm{Pd}\left(\mathrm{PPh}_{3}\right)_{4}(4 \mathrm{mg})$ were placed in a schlenk tube with a stir bar. The flask was evacuated and back-filled with argon free times before the addition of degassed toluene/diisopropylamine $(7: 3, \mathrm{v} / \mathrm{v}, 5.0 \mathrm{~mL})$ under an argon atmosphere. The tube was then sealed and heated at $60{ }^{\circ} \mathrm{C}$ for 3 days. The reaction mixture formed a gel, which was cooled to room temperature and dissipated in chloroform $(100 \mathrm{~mL})$. The insoluble part of the polymer was filtered and discarded. Only the soluble portion was applied to subsequent workup and characterizations. The combined organic layer was washed with sat. $\mathrm{NH}_{4} \mathrm{Cl}$ twice followed by brine, and then dried over anhydrous $\mathrm{Na}_{2} \mathrm{SO}_{4}$. The solvent was removed in vacuo. The residue was dissolved in a minimum amount of chloroform and reprecipitated in methanol. The precipitation was washed with hot methanol and acetone. The procedure was repeated three times to give a yellow solid (36\%). P2 and $\mathbf{P 3}$ were synthesized using the similar procedure for $\mathbf{P 1}$, except that the reaction mixture remained soluble throughout the polymerization reaction, no gel-like or insoluble product was formed and all the product was subjected to workup and characterizations. P1: ${ }^{1} \mathrm{H}$ NMR (300 MHz, $\left.\mathrm{CDCl}_{3}\right): 7.59$ (br, 4H), 7.04 (br, 4H), 6.57 (br, 8H), 4.00 (br, 8H), 1.94 (br, 8H), 1.55 (br, 8H), 0.88 (br, 16H), 0.40 (br, 12H). P2 (89 \%): ${ }^{1} \mathrm{H}$ NMR (500 MHz, $\left.\mathrm{CDCl}_{3}\right): 7.62$ (br, 4H), 7.43 (br, 8H), 7.15 (br, 4H), 7.02 (br, 8H), 6.37 (br, 4H), 5.82 (br, 4H), 4.25 (br, 4H), 4.07 (br, 4H), 2.13 (br, 8H), 1.62 (br, 8H), 1.03 (br, 8H), 0.88 (br, 8H), 0.37 (br, 12H). P3 (91\%): ${ }^{1} \mathrm{H}$ NMR (500 MHz, $\mathrm{CDCl}_{3}$ ): 7.66 (br, 4H), 7.42 (br, 4H), 7.33 (br, 4H), 
7.17 (br, 4H), 7.00 (br, 8H), 6.40 (br, 4H), 5.79 (br, 4H), 4.27 (br, 4H), 4.04 (br, 4H), $2.16(\mathrm{br}, 8 \mathrm{H}), 1.60$ (br, 8H), 1.05 (br, 8H), 0.89 (br, 8H), 0.41 (br, 12H).

Fluorescence Quenching. Fluorescence quenching experiments were carried out by micro-titration in solution in a fluorescence cuvette. In a typical titration procedure, $2.5 \mathrm{ml}$ of polymer solution was place in a 1-cm fluorescence cell. The fluorescence spectrum was recorded in the absence of quencher at room temperature. Then the fluorescent spectra were repeatedly acquired after microliter aliquots of a solution that contain the polymer at the same concentration and the quencher at a known concentration. The fluorescence quenching for the thin films was measured with the films placed in a closed cuvette where the quencher is contained and the vapor pressure of the quencher is pre-equilibrated prior to the placement of the thin film in the cell. The film in the cuvette was carefully positioned so that it was not in direct contact with the quencher but only exposed to the vapor. The intensity of the fluorescence was recorded with the lapse of time. 


\section{Stern-Volmer Plots}
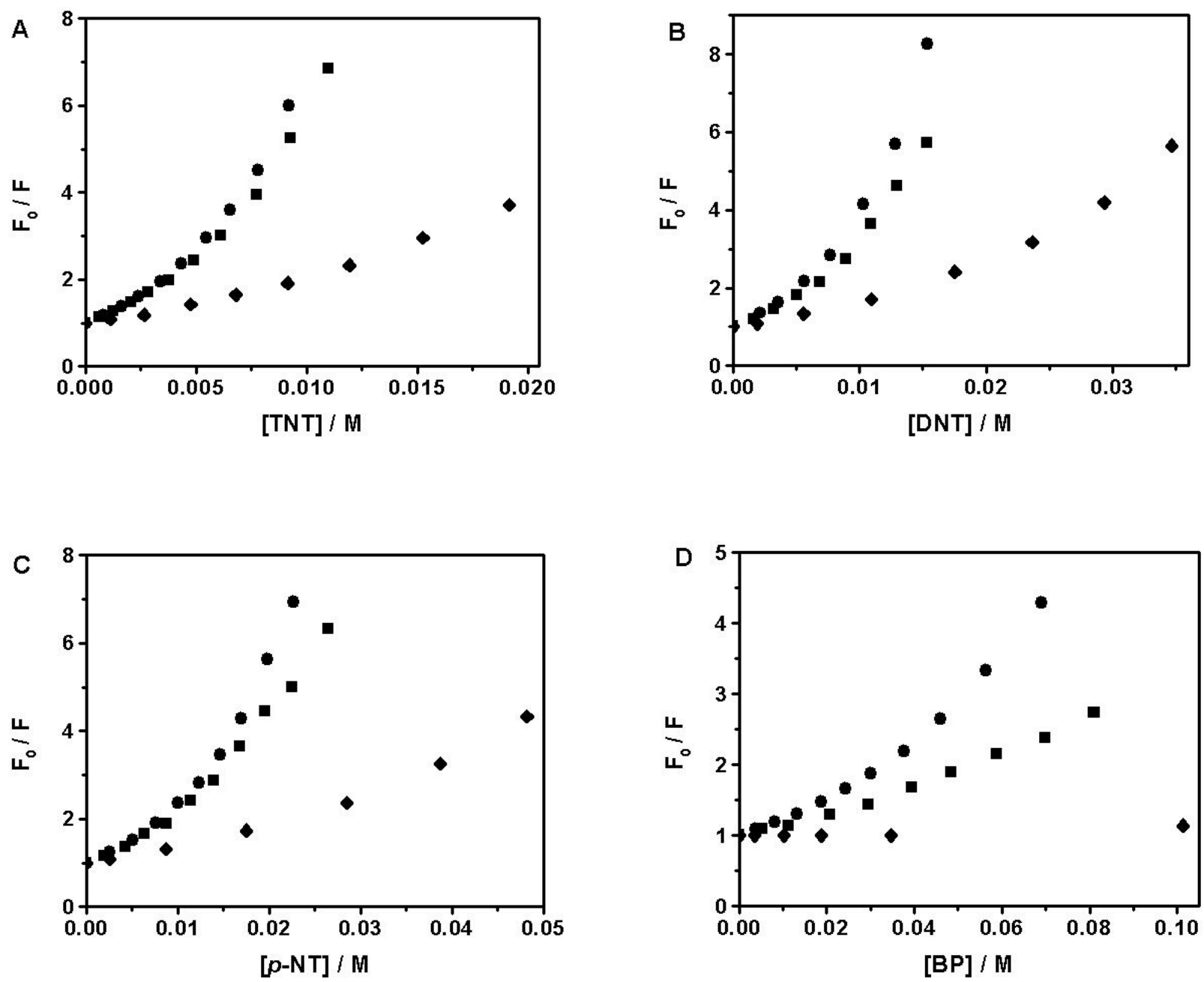

Figure S1. Stern-Volmer plots of P1 (square), P2 (circle) and $\mathbf{P 3}$ (diamond) in response to TNT (A), DNT (B), $p$-NT (C), and BP (D). 

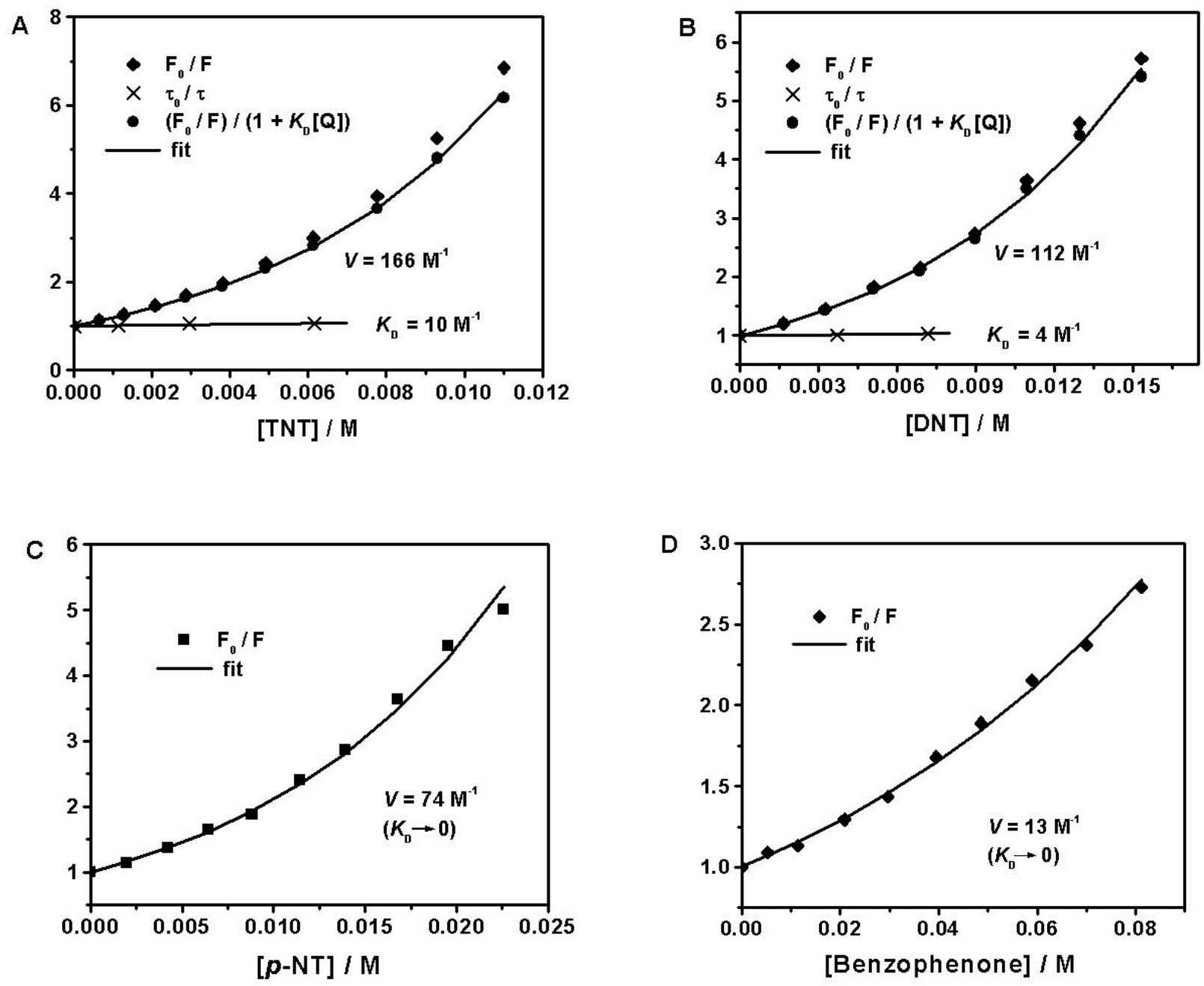

Figure S2. Stern-Volmer plots of $\mathbf{P 1}$ in response to different quenchers; the solid lines are best fit of the experimental data to Eqs. 2 and 3, with the determined quenching constants indicated. For $p$-NT and $\mathrm{BP}, K_{\mathrm{D}}$ was determined to be close to zero because no noticeable change in lifetime was detected. 
A
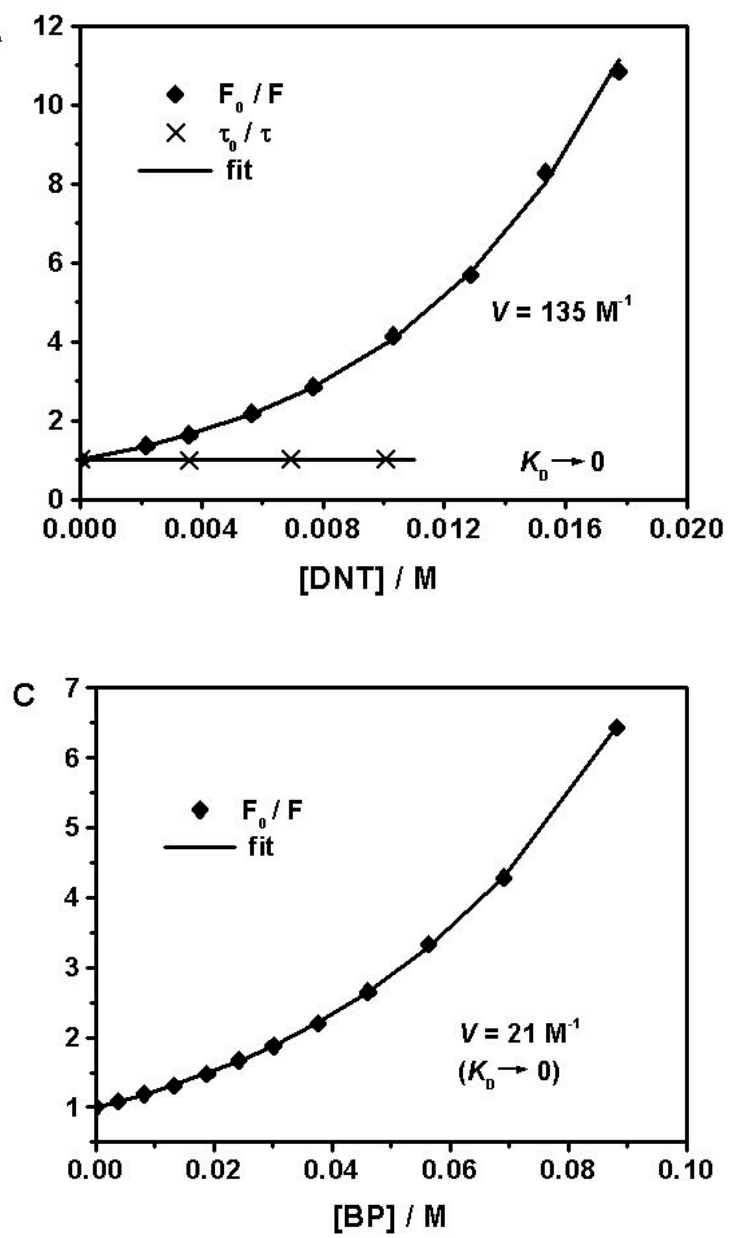

B

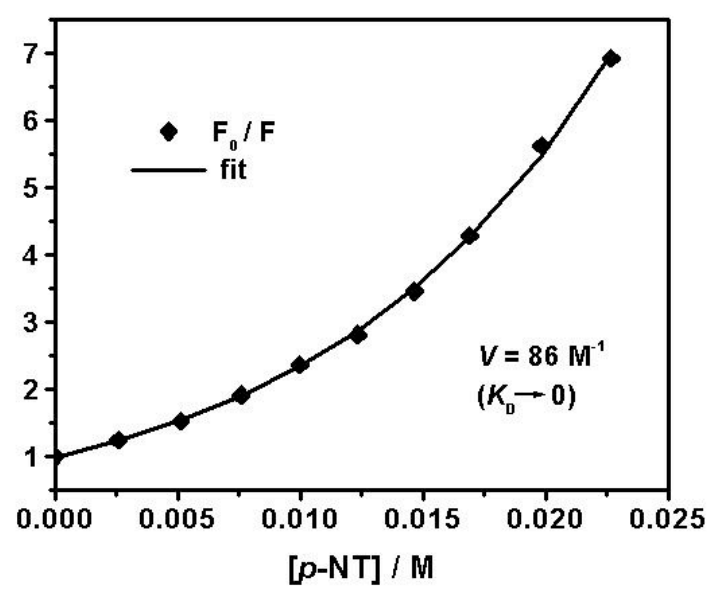

Figure S3. Stern-Volmer plots of $\mathbf{P 2}$ in response to different quenchers; the solid lines are best fit of the experimental data to Eqs. 2 and 3, with the determined quenching constants indicated. In all cases, $K_{\mathrm{D}}$ was determined to be close to zero because no noticeable change in lifetime was detected. 

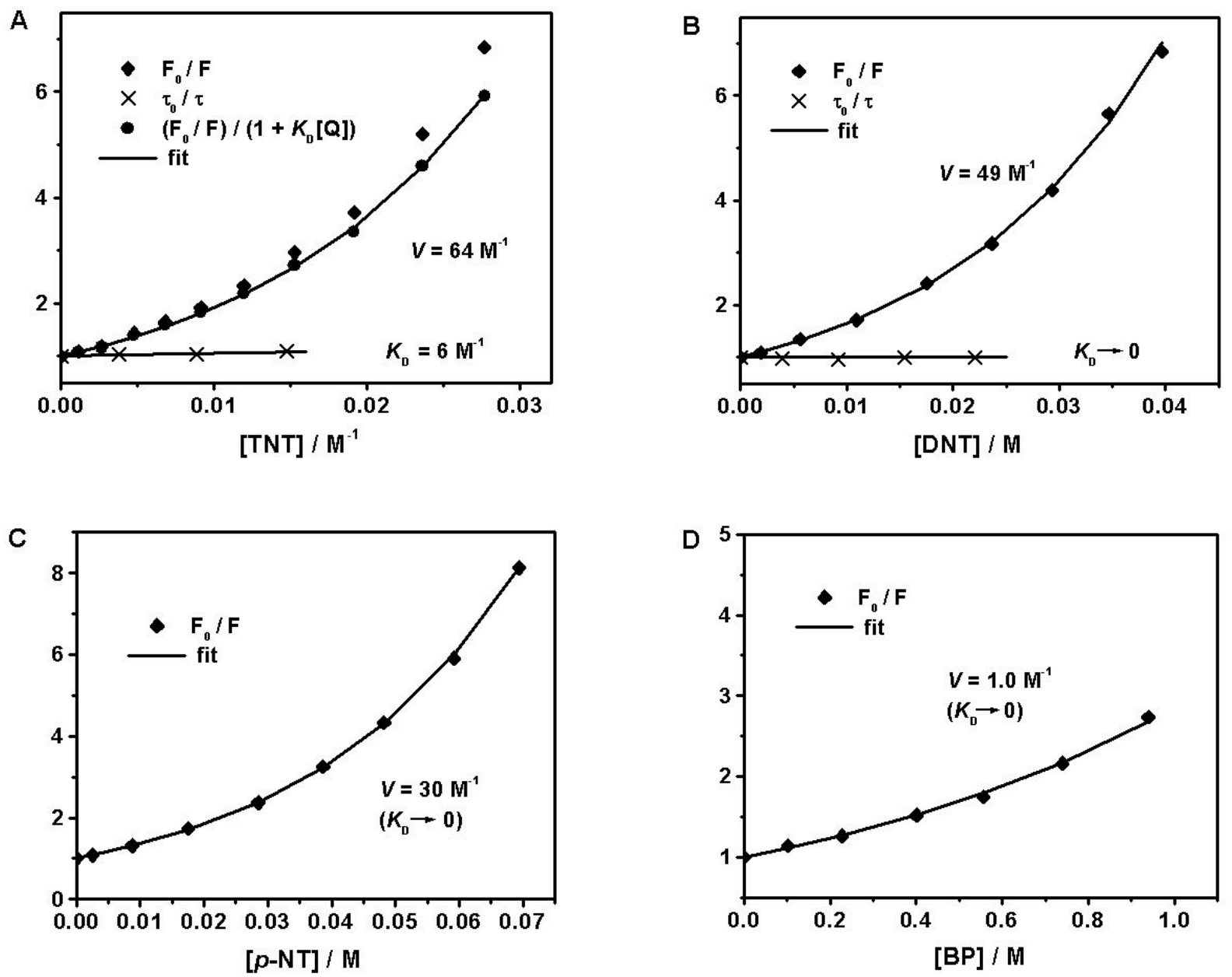

Figure S4. Stern-Volmer plots of P3 in response to different quenchers; the solid lines are best fit of the experimental data to Eqs. 2 and 3, with the determined quenching constants indicated. For DNT, $p$-NT and BP, $K_{\mathrm{D}}$ was determined to be close to zero because no noticeable change in lifetime was detected. 


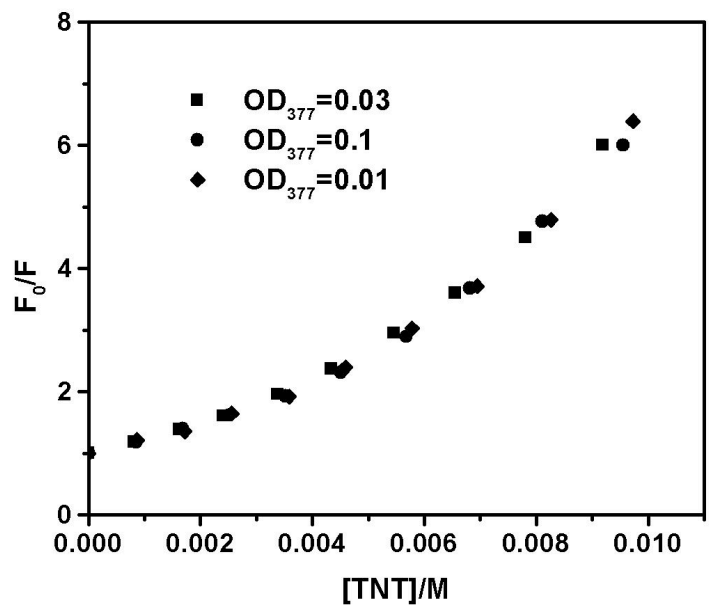

Figure S5. Stern-Volmer plots of $\mathbf{P 2}$ in response to TNT at varied polymer concentration in chloroform. The O.D.s of the polymer solution at $377 \mathrm{~nm}$ (excitation wavelength) are indicated.

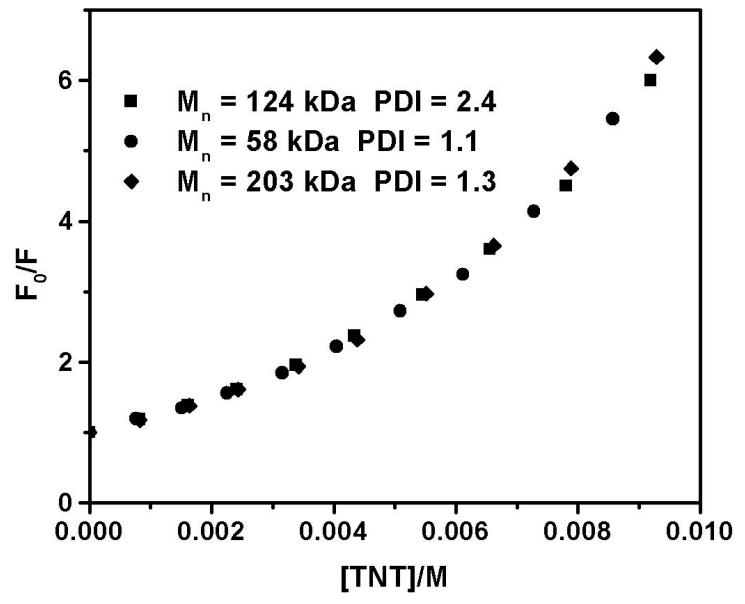

Figure S6. Stern-Volmer plots of $\mathbf{P 2}$ with varied MW (as indicated) in response to TNT. The narrow dispersed polymers were obtained through fractionation of the polydispersed polymer using SEC. 

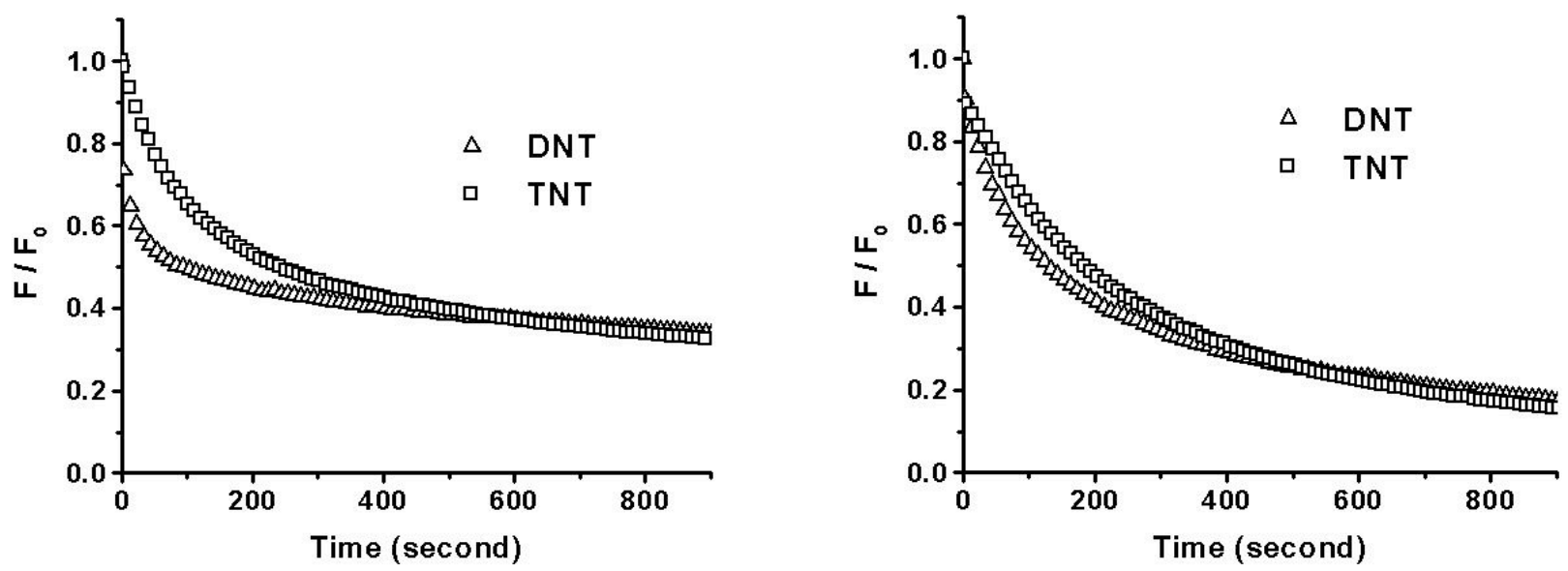

Figure S7. Fluorescence quenching of P1 (left) and P3 (right) in spin-cast thin films upon being exposed to TNT (square) and DNT (triangle) vapor.
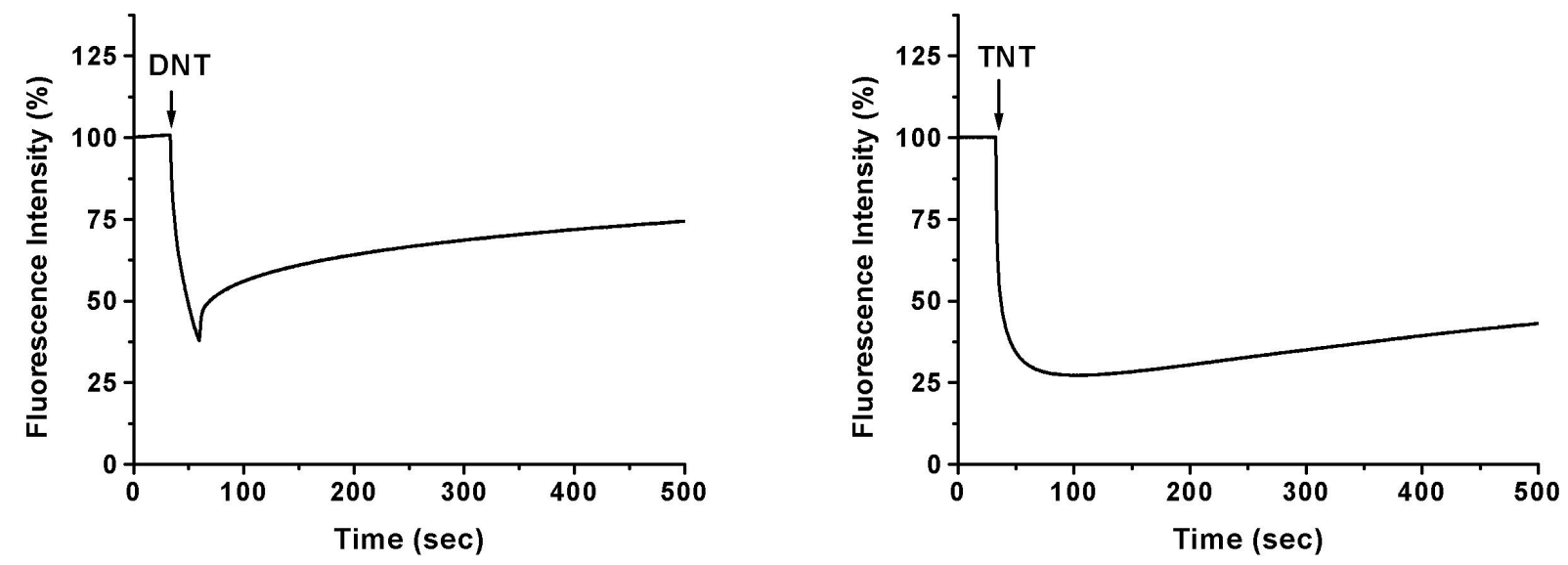

Figure S8. Real-time fluorescence intensity profiles (quenching followed by recovery) of $\mathbf{P 3}$ in response to DNT (left) or TNT vapor (right). The arrows indicate the points when quencher vapor was introduced. The exposure time for DNT is ca. 2 seconds and less than one second for TNT. 